\title{
Table des matières du tome second
}

\section{LA SCIENCE JURIDIQUE}

CHAPITRE VI. LA SCIENCE JURIDIQUE (Viktor KNAPP) 967

$\begin{array}{ll}\text { AVANT-PROPOS } & 969\end{array}$

SECTION I. CONSIDÉRATIONS D'ORDRE GÉNÉRAL 974

I. La notion des tendances de la recherche en science juridique 974

II. Notion de la science juridique 978

III. La géographie juridique 983

SECTION II. LES GRANDS COURANTS THÉORIQUES CONTEMPORAINS 994

I. Introduction (observations générales) 994

II. Pays occidentaux 998

1. Théories dualistes (le droit naturel) 999

2. Théories limitant l'objet de la connaissance juridique au droit en vigueur $\quad 1005$

3. Théories sociologiques 1008

III. Pays socialistes (la théorie marxiste-léniniste du droit) 1014

SECTION III. TENDANCES PRINCIPALES

I. Introduction (observations générales) 1024

II. Méthodologie 1024

1. L'application des méthodes sociologiques 1025

2. Le droit comparé 1027

(a) Problèmes généraux 1027

(b) Tendances actuelles 1034

3. L'étude de l'applicabilité au domaine de la science juridique des méthodes empruntées aux sciences exactes

(a) Problèmes généraux

(b) Tendances actuelles 
III. Histoire du droit

1. L'objet de l'histoire du droit

1049

2. Le droit romain

1050

3. Historia magistra vitae

IV. Le droit et l'homme

1052

1. Introduction (observations générales) 1052

2. Le droit au service de l'homme 1053

3. Ethnologie juridique 1058

V. Le droit et la pratique démocratique 1059

VI. Acculturation du droit 1062

1. L'acculturation du droit dans les pays ayant récemment accédé à l'indépendance

2. Problèmes spécifiques de l'acculturation du droit musulman

3. Problèmes spécifiques de l'acculturation du droit au Japon

4. Les enclaves indigènes

VII. Tendances actuelles intéressant le domaine des rapports internationaux

1. Le droit international et les problèmes actuels de la politique internationale

2. L'adaptation du droit interne aux normes du droit international

VIII. Tendances nouvelles dues à l'essor des sciences de la nature et des techniques permises par ces progrès scientifiques

1. Tendances actuelles intéressant le domaine du droit international et dues à l'essor des sciences de la nature: le droit spatial

2. Incidences et problèmes juridiques des greffes d'organes

EPILOGUE

1. Conclusions et perspectives

2. Liaisons interdisciplinaires

3. Epilogue de 1'Epilogue

I. Bibliographies juridiques 1100

II. Liste des ouvrages cités 
IV. LA PHILOSOPHIE (Paul RICOEUR)

AVANT-PROPOS: LA PHILOSOPHIE ET LES PHILOSOPHIES AUJOURD'HUI

SECTION I. L'HOMME ET SES SAVOIRS: PENSER

A. Philosophie de la logique

$\S 1$. Logique et mathématiques

1140

$\S 2$. La philosophie mathématique

$\S 3$. La philosophie de la logique: problèmes de fondement

$\S 4$. La logique des sciences

$\S 5$. Logique et ontologie dans la pensée indienne et japonaise contemporaine

B. Logique de la philosophie

Introduction

§1. Philosophie analytique: la tâche d'élucidation

$\S 2$. Le type analytique (suite): démarche transcendantale

$\S 3$. Philosophie synthétique: les systèmes non marxistes

$\S 4$. Le type synthétique (suite): logique et dialectique dans le matérialisme dialectique

$\S 5$. Logique de la philosophie et formalisation

$\S 6$. La signification philosophique de l'histoire de la philosophie

A. Epistémologie des sciences de la nature

$\S 1$. Loi et théorie

$\S 2$. La probabilité

$\S 3$. L'induction

$\S 4$. Logique de la découverte

B. Théorie de la réalité naturelle 1223

$\S 1$. Le référent du discours scientifique 1225

$\S 2$. Les catégories de la réalité $\quad 1230$

(a) Les notions d'événement et de temps 1230

(b) La notion d'espace 1232

(c) L'idée de cause 1234

§3. Les niveaux de la réalité 1238

(a) L'organisation biologique 1238

(b) La conscience 1241

$\S 4$. Le concept de réalité naturelle dans le matérialisme dialectique

§5. Philosophie de la nature 1257

§6. Langage et ontologie 1265

§ 7. Phénoménologie et ontologie 1273 
SECTION III. L'HOMME ET LA RÉALITÉ SOCIALE 1282

A. La logique de l'explication $\quad 1283$

$\S 1$. La logique de l'explication dans les sciences sociales 1283

(a) L'objet des sciences sociales 1283

(b) L'explication 1286

(c) Neutralité et valeur $\quad 1291$

(d) Rationalité 1293

e) Le problème distinctif de la connaissance dans les sciences sociales

§ 2. La logique de l'explication en psychologie 1297

a) Le behaviorisme 1297

b) Physicalisme ancien et nouveau $\quad 1304$

c) Structuralisme génétique 1306

§ 3. La psychanalyse et les sciences humaines 1309

§4. Epistémologie des sciences historiques 1318

a) Les philosophes de langue anglaise $\quad 1322$

b) La philosophie soviétique 1327

c) Les travaux de langue française $\quad 1333$

B. Philosophie sociale et politique 1337

$\begin{array}{ll}\text { Introduction } & 1337\end{array}$

§ 1. Philosophie du droit et philosophie politique 1338

a) Philosophie du droit 1338

b) Philosophie politique 1346

$\S 2$. Le matérialisme historique $\quad 1356$

a) La théorie du reflet $\quad 1357$

b) Le rapport de la conscience individuelle et de la conscience sociale 1358

$\S 3$. L'homme et la réalité sociale dans une anthropologie philosophique

a) Liberté et institutions $\quad 1366$

b) Pouvoir et violence $\quad 1372$

c) Violence et discours $\quad 1375$

CHAPITRE VIII. LE LANGAGE, L'ACTION, L'HUMANISME 1379

SECTION IV. L'HOMME ET LE LANGAGE 1381

A. Epistémologie de la linguistique 1381

$\S 1$. Linguistique structurale et linguistique transformationnelle 1381

$\S 2$. Linguistique et sémiologie 1388

$\S 3$. Linguistique et sciences humaines $\quad 1390$

$\S 4$. Linguistique et sciences de la nature 1393

B. Philosophies du langage $\quad 1395$

Introduction $\quad 1395$

$\S 1$. La conception « analytique » 1397

$\S 2$. Phénoménologie du langage 1403 
§3. Le marxisme et les problèmes philosophiques du langage 1406

$\S 4$. Le structuralisme philosophique 1413

$\S 5$. Le courant « herméneutique »: l'interprétation du langage 1418

$\S 6$. Langage et ontologie selon les penseurs récents de l'Orient 1424

SECTION V. L'HOMME ET L'ACTION

A. Théorie du discours pratique

$\S 1$. Théorie de la décision et des jeux

$\S 2$. Logique du discours pratique: logique déontique 1438

$\S 3$. Le raisonnement pratique et la théorie de l'argumentation 1446

B. Les philosophies de l'action

$\S 1$. La philosophie "analytique " et les problèmes de l'action humaine

§ 2. La liberté et la philosophie des "valeurs »

$\S 3$. Théorie et pratique dans la philosophie du marxisme

SECTION VI. L'HOMME ET LE FONDEMENT DE L'HUMANISME

A. L'homme selon les pensées de l'Orient

B. L'homme selon les pensées de l'Occident

$\S 1$. La conception analytique de l'homme et de l'humanisme et « the concept of mind"

$\S 2$. Le marxisme et la discussion sur l'humanisme

$\S 3$. La discussion sur l'humanisme dans la philosophie « continentale $"$

C. La philosophie de la religion

Introduction

$\S 1$. L'ontologie-théologie: tradition et renouveau

$\S 2$. La critique de la religion dans la " philosophie analytique "

§3. La philosophie de la religion à l'école des sciences de l'homme

§4. " Jeux de langage » et « actes de discours » religieux

$\S 5$. L'herméneutique du langage religieux

Conclusion: l'humanisme à la recherche de son fondement 



\section{La science juridique}


